\title{
Under pressure - Der Stellenwert des zentralen Venendrucks in der modernen Intensivmedizin
}

\author{
Michael Heßler, Philip-Helge Arnemann, Christian Ertmer
}

Die Steuerung einer Volumentherapie einzig am zentralen Venendruck ist obsolet und wahrscheinlich sogar schädlich. ZVD und zentrale Venenpulskurve liefern aber wichtige Informationen. Nur zur ZVD-Messung sollte man jedoch keinen ZVK anlegen.

\section{Einleitung}

Als zentraler Venendruck (ZVD) wird der Druck in der oberen Hohlvene (V. cava superior) sowie im rechten Vorhof bezeichnet. Dieser entspricht annähernd dem Füllungsdruck des rechten Ventrikels. Der ZVD wird auf Intensivstationen in der Regel mit einem invasiven Messverfahren bestimmt. Wird der ZVD zur Beurteilung pathologischer Zustände herangezogen, muss bedacht werden, dass multiple Faktoren Einfluss auf ihn ausüben (z.B. Funktion des rechten Ventrikels, Flüssigkeitshaushalt, intrathorakale Druckverhältnisse und Venentonus) [1]. In den zurückliegenden Jahren wurde eine teils emotionale Diskussion über die Bedeutung des ZVD als Surrogatparameter der Vorlast oder zur Beurteilung der Volumenreagibilität geführt [2-4]. Diese Diskussion hat dazu geführt, dass der Parameter ZVD selbst unter Druck geraten ist und sein Image gelitten hat. Vorausgesetzt der ZVD wird korrekt bestimmt, kann man durch die Messung des ZVD bzw. die Beurteilung der Druckkurve jedoch auch heute noch relevante Informationen über den Patienten gewinnen, unabhängig von seiner Eignung als Parameter der Volumenreagibilität. Die vorliegende Übersichtsarbeit diskutiert anhand der aktuellen Evidenz den Stellenwert des ZVD in der Intensivmedizin.

\section{Die Bestimmung des zentralen Venendrucks}

Historische Entwicklung. Etwa 100 Jahre nach der Beschreibung des Blutkreislaufs durch den englischen Arzt William Harvey (1578-1657) und der Veröffentlichung seines epochalen Werks „De Motu Cordis“ im Jahr 1628 wurde erstmals über eine erfolgreiche Katheterisierung einer zentralen Vene berichtet. Der englische Geistliche Stephen Hales platzierte bei einem liegenden Pferd ein dünnes Metallröhrchen in der linken Jugularvene und verband dieses mit einem aufrechten Glasrohr. Mit dieser Versuchsanordnung konnte Hales den Venendruck des Pferdes messen [5,6]. Die ersten zentralvenösen Kanülierungen beim Menschen wurden von Bleichröder im Jahr 1905 durchgeführt [7]. Vermehrtes Interesse jedoch erhielt seine Methode erst mit neuen medizinischen Erkenntnissen, die es notwendig machten, Medikamente zentralvenös geben zu können. Pionier auf diesem Gebiet war der Deutsche Werner Forssmann, der zentralvenöse Katheter ab den 1920er-Jahren zuerst im Selbstversuch über die Fossa cubiti einführte und schon damals Röntgenstrahlen zur Lagekontrolle der Katheter verwendete. Für seine Arbeiten zur zentralvenösen Kanülierung wurde Forssmann schließlich 1956 zusammen mit André Cournand und Dickinson Richards mit dem Nobelpreis für Medizin ausgezeichnet [6]. 
Zugangswege. In der Regel wird der ZVD invasiv über einen zentralvenösen Katheter (ZVK) bestimmt. Zur Bestimmung des ZVD sollte die Spitze des ZVK am Übergang der V. cava superior zum rechten Vorhof, in der V. cava superior oder im hohen rechten Vorhof (mit ausreichendem Abstand zur Trikuspidalklappe) platziert werden [1]. Hier befindet sich der hydrostatische Nullpunkt, auf den sich die Druckmessung bezieht (s.u.). ZVK können über verschiedene Venen zur Messung des ZVD eingebracht werden (u.a. über die V. axillaris, V. subclavia, V. femoralis oder die V. brachialis).

Der Zugangsweg über die rechte V. jugularis interna, beschrieben von English et al. im Jahr 1969 [8], wird in der Klinik häufig verwendet, da der Katheter durch den fast geraden, klappenlosen Weg zum Herzen meist erfolgreich positioniert werden kann (über $90 \%$ bei Erwachsenen und Kindern) und der Punktionsort am Hals den Zugriff auf den Katheter erleichtert [1]. Die Mehrzahl zentralvenöser Kanülierungen wird in Seldinger-Technik durchgeführt $[9,10]$. Liegen relative Kontraindikationen zur Punktion der V. jugularis interna einer Seite vor, kann man erwägen, das Gefäß der Gegenseite zu punktieren. Dabei muss man jedoch bedenken, dass bei Punktion der V. jugularis interna sinistra durch die schräge Einmündung der V. brachiocephalica sinistra in die V. cava superior die Gefäßwand leichter perforiert werden kann. Eine Punktion der linken V. jugularis kann zudem bei proximaler Punktion zur Verletzung des Ductus thoracicus führen [1]. Zunehmend werden im klinischen Alltag zentralvenöse Zugänge sonografisch gesteuert gelegt.

Im Vergleich zur Landmarken-Methode ist die sonografische Methode häufiger erfolgreich, entwickelt weniger Komplikationen und gelingt öfter beim ersten Punktionsversuch [11, 12].

Lagekontrolle. Nach dem Einbringen eines ZVK ist eine Lagekontrolle notwendig, wozu man meist eine Ableitung eines EKG über den Katheter (AlphacardMethode) [13], ein Thoraxröntgenbild oder eine transthorakale Echokardiografie heranzieht [14]. Eine korrekte Platzierung ist Voraussetzung für valide ZVDMesswerte; mit einer Thoraxübersichtsaufnahme oder der transthorakalen Echokardiografie kann man gleichzeitig auch einige Komplikationen ausschließen.

Messprinzip. Auch heute noch wird das Grundprinzip angewendet, welches schon Hales zur Bestimmung des Venendrucks nutzte. Der Messung des ZVD liegt dabei das Prinzip der kommunizierenden Röhren zugrunde: Dabei wird ein in der V. cava superior platzierter
Katheter mit einem bettseitig angebrachten, wassergefüllten Rohr verbunden, das zur Atmosphäre hin geöffnet ist. Der Wasserpegel in dem Glasrohr nimmt so weit ab, bis der in der V. cava superior herrschende Druck dem hydrostatischen Druck der Wassersäule entspricht. An einer Skala kann man nun den Venendruck ablesen, der folglich in Zentimeter Wassersäule $\left(\mathrm{cmH}_{2} \mathrm{O}\right)$ gemessen wird [15].

Heute führt man die hydrostatische Messung des ZVD in der Regel über einen Drucksensor (Transducer) durch, der über einen Schlauch mit einem zentralen Venenkatheter verbunden ist. Kalibriert und dargestellt wird das Signal des Drucksensors über einen Monitor. Der ZVD wird heute - dem arteriellen Blutdruck angepasst - gewöhnlich in Millimeter Quecksilbersäule ( $\mathrm{mmHg}$ ) angegeben.

Praktischer Messvorgang. Der erste Schritt zur Messung des ZVD nach dem Anschluss des gespülten Messsystems ist der „Nullabgleich“ gegen den atmosphärischen Druck durch Öffnen eines Dreiwegehahns in der Nähe des Drucksensors. Um reproduzierbare Messergebnisse zu erhalten, ist es wichtig, dass der Drucksensor auf Höhe einer horizontalen Ebene durch die Trikuspidalklappe angebracht ist [15]. Guyton et al. zeigten, dass an diesem Punkt der Druck im Venensystem weitgehend konstant und relativ unabhängig von der Körperlage ist [16]. Durch Anbringen des Drucksensors auf dieser Höhe kann der Einfluss des hydrostatischen Drucks auf die Messergebnisse minimiert werden. Um diesen „Nullpunkt“ in der Klinik näherungsweise einzustellen, sollte der Drucksensor ungefähr auf Höhe von vier Fünfteln des Thoraxdurchmessers (gemessen von der Rückseite des Patienten) in Rückenlage angebracht werden [17]. Je nach klinischer Situation muss man bedenken, dass nicht jeder Patient zur Bestimmung des Referenzpunkts und zur Messung des ZVD in Rückenlage gebracht werden kann. In jedem Fall wird ein zu hoch angebrachter Drucksensor falsch niedrige ZVD-Werte messen, während ein in Bezug auf den Nullpunkt zu niedrig angebrachter Drucksensor zu falsch hohen Messwerten führt. Das Messergebnis ändert sich pro Zentimeter Abweichung vom Nullpunkt um ungefähr $\pm 0,75 \mathrm{mmHg}\left(1 \mathrm{cmH}_{2} \mathrm{O}\right)$.

Wichtig ist, dass bei Änderungen der Patientenposition auch die Höhe des Drucksensors verändert wird.

Obwohl eine falsche Einstellung der Position des Drucksensors entscheidenden Einfluss auf den Wert des ZVD hat, wird diese in der Klinik häufig falsch 
gewählt $[18,19]$. Selbst die Benutzung eines Linienlasers zur Identifizierung anatomischer Landmarken konnte die Variation der Position des Drucksensors nicht signifikant vermindern. Unter Umständen sind eine klinikweite Standardisierung und Mitarbeiterschulungen eine Möglichkeit, diese Variation zu minimieren [19].

Einfluss des intrathorakalen Drucks. Für eine korrekte Messung des ZVD müssen die Druckschwankungen im Thorax während eines Atemzyklus unter Spontanatmung beachtet werden. Der vom Drucksensor gemessene Druckwert wird beeinflusst von der transmuralen Druckdifferenz $\left(P_{\text {transmural }}\right)$ in der oberen Hohlvene. $P_{\text {transmural }}$ ist die Differenz zwischen intraund extravasalem Druck (d.h. Differenz zwischen ZVD und intrathorakalem Druck $P_{\text {intrathorakal }}$ [20]. Während der Inspiration sinkt $P_{\text {intrathorakal }}$, erst endexspiratorisch nähert sich $P_{\text {intrathorakal }}$ dem atmosphärischen Druck an, gegen den der Drucksensor abgeglichen ist. Endexspiratorisch entspricht daher $\mathrm{P}_{\text {transmural }}$ weitgehend dem ZVD. Den ZVD muss man daher endexspiratorisch bestimmen [21]. Dies ist auch der Grund, warum der auf Monitoren meist angezeigte mittlere ZVD formal gesehen nicht korrekt ist [15]. Ist der Patient beatmet und wird ein positiver endexspiratorischer Druck (PEEP) verwendet oder erzeugt der Patient im Zuge einer Bronchialobstruktion einen „Auto-PEEP“, kann der ZVD aufgrund der Steigerung des intrathorakalen Drucks überschätzt werden. Bei niedrigem PEEP kann dieser Effekt häufig vernachlässigt werden [21].

Die Messung des ZVD mit einem wassergefüllten Rohr zeigt ebenfalls atemsynchrone Schwankungen der Wassersäule infolge der intrathorakalen Druckänderungen. Aufgrund der Trägheit der Wassersäule kann der endexspiratorische ZVD jedoch nicht korrekt abgelesen werden. Beim spontan atmenden Patienten sollte man den ZVD während der Systole ablesen. Unter Beatmung entspricht der diastolische endexspiratorische Wert dem ZVD - aufgrund der Modulation durch die intrathorakalen Druckschwankungen.

In der Literatur wird der Normwert des ZVD mit

$4-16 \mathrm{cmH}_{2} \mathrm{O}(3-12 \mathrm{mmHg})$, bzw. in SI-Einheiten

0,4-1,6 kPa angegeben (Umrechnungsfaktoren:

$1 \mathrm{mmHg}=$ ca. $1,36 \mathrm{cmH}_{2} \mathrm{O} ; 1 \mathrm{mmHg}=$ ca. $0,13 \mathrm{kPa}$ )

[15].

Derzeitiger Goldstandard zur Bestimmung des ZVD ist die invasive Messung über einen ZVK im oberen Hohlvenensystem, welche ohne größeren Aufwand auch die wiederholte oder kontinuierliche Messung ermöglicht.
Intensivmedizinische Patienten haben etwa in der Hälfte der Fälle einen ZVK [22]. Der ZVK dient zur Applikation von Medikamenten, zur Unterstützung der hämodynamischen Überwachung mit Messung der zentralvenösen Sauerstoffsättigung und darüber hinaus als sicherer Zugang bei schlechtem Zustand der peripheren Venen.

Nicht invasive Messmethoden. Kürzlich wurden in der Literatur vermehrt nicht invasive Methoden zur Abschätzung des ZVD beschrieben, da das Anlegen eines ZVK mit Risiken und Komplikationen verbunden ist. Nicht invasive Methoden zur Abschätzung des ZVD während der körperlichen Untersuchung sind u. a. die Bestimmung des Jugularvenendrucks, der „Peripheralvenous-collapse“-Test sowie die sonografische Darstellung der V. cava inferior [23]. Wie viele klinische Untersuchungsmethoden ist das Ergebnis interpersonellen Schwankungen zwischen den Untersuchern unterworfen, welche auf Erfahrungen und Fertigkeiten beruhen, und es hängt von der körperlichen Konstitution des Patienten ab. Beispielsweise kann eine starke Adipositas die nicht invasive Abschätzung des ZVD unmöglich machen $[23,24]$. Trotz ihrer methodologischen Einschränkungen können nicht invasive Methoden u.a. in Notfallsituationen oder Ambulanzen hilfreich sein zur schnellen Abschätzung des ZVD [24]. Auch bei Neugeborenen, bei denen die zentralvenöse Punktion manchmal schwierig ist oder vermieden werden soll, können nicht invasive sonografische Messverfahren nützlich sein [25].

Fehlerquellen bei der ZVD-Messung. Bei kaum einem anderen klinischen Parameter ist die korrekte Bestimmung so entscheidend für ein valides Messergebnis wie beim ZVD. Ein Unterschied im systolischen Blutdruck von $\pm 10 \mathrm{mmHg}$ (durch ungenaue Messmethodik) wird in vielen Fällen zu vernachlässigen sein, während ein solcher Unterschied bei der Messung des ZVD gravierende Bedeutung hat.

Den ZVD muss man, v.a. bei plötzlichen Änderungen, zwingend unter Kenntnis möglicher Fehlerquellen und im klinischen Kontext des Patienten bewerten.

Häufige Fehlerquellen bei der ZVD-Messung sind in Tab. 1 aufgeführt. 


\section{Tabelle 1}

Fehlermöglichkeiten bei der Bestimmung des zentralen Venendrucks (mod. nach [15]).

\begin{tabular}{|c|c|}
\hline Fehlerquelle & Mögliche Ursachen \\
\hline Nullpunkt & $\begin{array}{l}\text { - kein Referenzpunkt festgelegt } \\
\text { - Drucksensor nicht gegen Nullpunkt abgeglichen } \\
\text { - Drucksensor zu hoch oder zu tief } \\
\text { - Positionsveränderung des Patienten gegenüber dem } \\
\quad \text { Drucksensor }\end{array}$ \\
\hline Katheter & $\begin{array}{l}\text { - Drucksensorsystem und Katheter nicht durchgespült } \\
\text { - intravasale Fehllage (Katheter nicht in V. cava superior } \\
\text { oder im rechten Vorhof) } \\
\text { - Wandkontakt } \\
\text { - extravasale Fehllage }\end{array}$ \\
\hline $\begin{array}{l}\text { Druckerhöhung im } \\
\text { Thorax }\end{array}$ & $\begin{array}{l}\text { - mechanische Beatmung } \\
\text { - Pneumothorax } \\
\text { - Pressen oder Husten beim Messvorgang } \\
\text { - starke Kräfte bei Spontanatmung (z. B. Asthma, inspi- } \\
\text { ratorische Obstruktion) }\end{array}$ \\
\hline technische Ursachen & $\begin{array}{l}\text { - Luft im Messsystem } \\
\text { - Messabweichung des Drucksensors } \\
\text { - Kompression (Abknicken) eines Druckschlauchs des } \\
\text { Messsystems }\end{array}$ \\
\hline
\end{tabular}

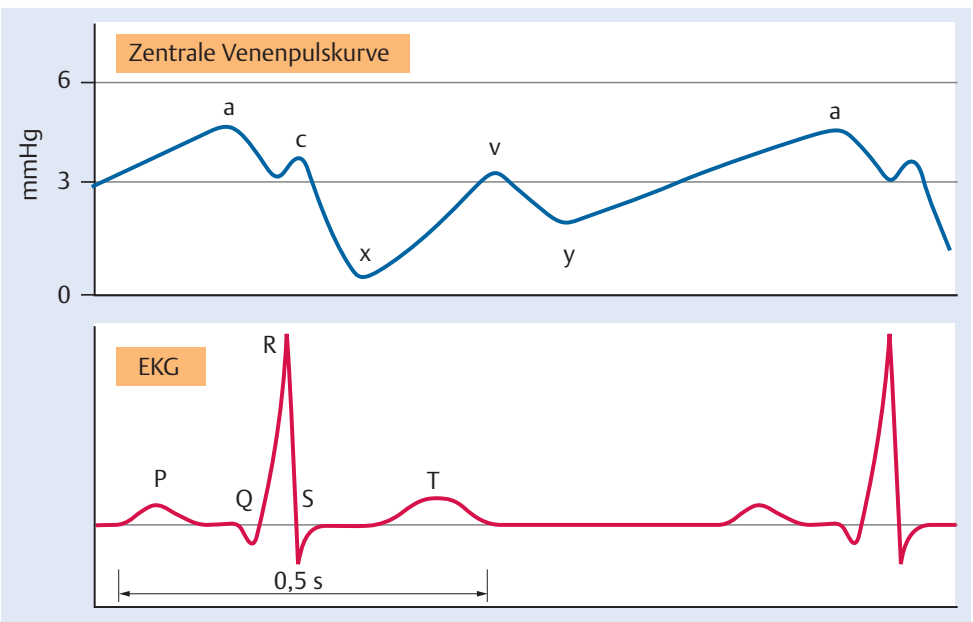

Abb. 1 Zentrale Venenpulskurve (mod. nach [20, 26]). Die Abbildung zeigt schematisch die vom Herzzyklus abhängigen Schwankungen der zentralen Venenpulskurve. Zur Orientierung am zeitlichen Ablauf ist unterhalb der Venenpulskurve ein EKG skizziert. Die Druckschwankungen der Venenpulskurve lassen sich folgenden Vorgängen zuordnen: a Kontraktion des rechten Vorhofs

c Kontraktion des rechten Ventrikels mit Vorwölbung der Trikuspidalklappe in den rechten Vorhof

x Druckabnahme durch Verschiebung der Ventilebene

v passive Füllung des rechten Vorhofs bei geschlossener Trikuspidalklappe

y Bluteinstrom in den Ventrikel

\section{Von der Physiologie zur Entwicklung von pathophysio- logischen Konzepten}

\section{Venenpulskurve}

Wird der ZVD über die Zeit auf einem Monitor aufgezeichnet, erkennt man atmungsabhängige Schwankungen durch intrathorakale Druckänderungen. Darüber hinaus beeinflusst auch die Pulsation des Herzens den ZVD. Die Veränderung über die Zeit spiegelt die Venenpulskurve wider. Der physiologische Verlauf der zentralen Venenpulskurve ist typischerweise durch Wellen und Senken gekennzeichnet (Abb. 1).

Die erste Welle (a-Welle) der Venenpulskurve wird durch die Kontraktion des rechten Vorhofs ausgelöst und folgt der P-Welle im EKG. Während der Kontraktion des rechten Ventrikels wölbt sich die Trikuspidalklappe in den rechten Vorhof. Diese Drucksteigerung wird als c-Welle bezeichnet. Im klinischen Alltag ist die c-Welle oft nur schwer zu identifizieren. Die auffällige $\mathrm{x}$-Senke folgt aus der Verschiebung der Ventilebene zur Herzspitze in der Austreibungsphase des Herzzyklus. Die v-Welle der Venenpulskurve ergibt sich aus der passiven Füllung des Vorhofs während der späten Systole und frühen Diastole mit Entspannung des Ventrikels, während die Segel der Trikuspidalklappe noch geschlossen sind (isovolumetrische Relaxation), und erscheint synchron mit der T-Welle. Dem Öffnen der Trikuspidalklappe und der Füllung des rechten Ventrikels entspricht die charakteristische Druckabnahme nach der v-Welle, welche als y-Senke bezeichnet wird $[20,26,27]$. Ein abweichender Verlauf der Venenpulskurve kann durch verschiedene pathologische Ursachen bedingt sein (Abb.3). Jedoch können auch Fehllagen des ZVK zur Abweichung von der typischen Venenpulskurve führen: Ein Wandkontakt der ZVKSpitze kann zu einem charakteristischen Treppenmuster der zentralen Venenpulskurve führen [15].

\section{Einflussfaktoren auf den ZVD}

Der ZVD wird von vielen Faktoren beeinflusst, so etwa durch den transmuralen Druck im Thorax (u.a. intrathorakaler Druck und PEEP), die Compliance der Gefäße, den Gefäßwiderstand, das Blutvolumen sowie die Pumpfunktion des Herzens [18]. Kreislaufphysiologische Modelle helfen zu erklären, welchen Einfluss einzelne Variablen auf den ZVD ausüben. 
Elastizität und Kapazität des venösen Systems. Gauer beschreibt, in Anlehnung an das Kreislaufmodell von Weber, den Kreislauf als ein System kommunizierender elastischer Röhren. Bei Kreislaufstillstand herrscht in allen Gefäßabschnitten der „statische“ Blutdruck. Dieser hängt einerseits ab vom Blutvolumen, andererseits von der Volumenelastizität des Gefäßsystems. Mit Beginn der Pumpfunktion des Herzens steigt der Blutdruck auf arterieller Seite, während im venösen System der Blutdruck sinkt, bis sich ein Gleichgewicht zwischen Blutzustrom und Abstrom im arteriellen System eingestellt hat. Angenommen, beide Kompartimente des Gefäßsystems weisen die gleiche Volumenelastizität auf, so würde der Blutdruck um den gleichen Betrag sinken, wie der Druck im arteriellen System erhöht würde. In der Tat weist das venöse System eine ungefähr 200-mal größere Volumenelastizität als das arterielle System auf und enthält etwa $85 \%$ des Blutvolumens (daher auch Kapazitätssystem genannt). Die Druckänderung fällt daher im venösen System wesentlich geringer aus als im arteriellen System. Der ZVD des aktiven Kreislaufs unterscheidet sich daher vom statischen Druck nur unwesentlich - im Vergleich zur Differenz zwischen arteriellem und statischem Druck [28].

Der Vergleich des Gefäßsystems mit einem elastischen Gefäß trifft zu, solange die Weitbarkeit (Kapazität) des Gefäßsystems und die Herzfunktion konstant bleiben. Blutverlust oder Transfusion bewirken dann ein Sinken oder Steigen des ZVD. Gauer selbst wies diesen Zusammenhang für moderate Volumenänderungen nach (Hämorrhagie 6,5 ml/kgKG und [Re-]Transfusion $8,1 \mathrm{ml} / \mathrm{kgKG}$ ) [29]. Zu dieser nach Gauer ,exakten Beziehung“ bemerkt er selbst, dass diese nur gilt, wenn sich die glatten Gefäßmuskelzellen der kapazitiven Gefäße weder aktiv reflektorisch kontrahieren noch entspannen. Änderungen von $\pm 10-15 \%$ des Blutvolumens würden so nicht zu einer Änderung der potenziellen Kapazität des Kreislaufsystems führen [28]. Experimentell zeigte Gauer jedoch, dass die Volumenelastizität des Gefäßsystems z.B. durch Vasopressoren (Noradrenalin) beeinflusst werden kann [30]. Dies ist ein Grund dafür, warum unter Stress der venöse Druck keine lineare Funktion des entnommenen Volumens darstellt [29].

Mittlerer Füllungsdruck. Im anglo-amerikanischen Sprachraum prägte v.a. Arthur Guyton das Verständnis vom Blutkreislauf. Abweichend vom kardiozentrischen Kreislaufmodell Starlings, welches das Herz als treibende Kraft im Blutkreislauf ansieht [31], formulierte Guyton ab den 1950er-Jahren sein Modell des Kreislaufs, welches sich auf den venösen Rückstrom zum
Herzen konzentriert [32, 33]. Analog zum Begriff des statischen Blutdrucks führt Guyton den Begriff des mittleren Füllungsdrucks des Kreislaufs ein („mean circulatory filling pressure“, $\mathrm{P}_{\mathrm{ms}}$ ), der dem ausgeglichenen Druck im Gefäßsystem bei Kreislaufstillstand entspricht. $P_{\mathrm{ms}}$ wird aufgebaut aus den Wechselwirkungen von (elastischen) Gefäßwänden und dem Blutvolumen. Treibende Kraft für den venösen Rückstrom ist nach Guytons Modell der $\mathrm{P}_{\mathrm{ms}}$, dem der Druck im rechten Vorhof entgegensteht oder anders: Der venöse Rückstrom zum Herzen ist abhängig vom Gradienten zwischen $P_{m s}$ und dem Druck im rechten Vorhof (d.h. dem ZVD). Neben dem ZVD wirkt zusätzlich der Gefäßwiderstand dem venösen Rückstrom entgegen (Abb.4). Der Zusammenhang zwischen $\mathrm{P}_{\mathrm{ms}}$, ZVD und Gefäßwiderstand kann in Analogie zum Ohm-Gesetz geschrieben werden als:

venöser Rückstrom $=\frac{P_{m s}-Z V D}{\text { Gefäßwiderstand }}$

Guytons Kreislaufmodell wird von vielen Autoren akzeptiert [33-37], allerdings ist seine Theorie nicht unumstritten [38, 39]. Im Zusammenhang mit Guytons Kreislaufmodell wird häufig angenommen, dass der venöse Rückstrom das Herzzeitvolumen kontrolliert. Wichtig ist, dass im intakten Kreislauf der venöse Rückstrom dem Herzzeitvolumen entsprechen muss. Änderungen können daher immer nur vorübergehender Natur sein (Frank-Starling-Mechanismus), bis sich ein neues Fließgleichgewicht eingestellt hat (Abb.4) [40].

Intrathorakale Druckschwankungen. Die folgenden Faktoren beeinflussen den ZVD klinisch relevant. Druckschwankungen im Thorax während der (Be-)Atmung üben einen fortwährenden Einfluss auf den ZVD aus, typischerweise erkennbar an den Undulationen der zentralen Venenpulskurve auf einem Monitor. Unter spontaner Inspiration sinkt beispielsweise der intrathorakale Druck, während der intraabdominale Druck ansteigt. Der vergrößerte Druckgradient zwischen abdominalen und zentralen Venen unterstützt den Blutrückstrom zum Herzen. Die Veränderung der intrathorakalen Drücke sorgt dafür, dass man den ZVD endexspiratorisch bestimmen sollte (s.o.).

Blutvolumen und Venentonus. In einem bestimmten Bereich reagiert der ZVD auch auf eine Zu- oder Abnahme des Blutvolumens (s.o.). Zur Abnahme des ZVD führt u.a. eine Hämorrhagie oder Dehydratation. Auf der anderen Seite lässt eine Transfusion oder 
Abb. 2 Beeinflussung des zentralen Venendrucks (Erläuterungen siehe Text). $\mathrm{P}_{\mathrm{ms}}$ : mittlerer Füllungsdruck des Kreislaufs; VR: venöser Rückfluss zum Herzen; ZVD: zentraler Venendruck.

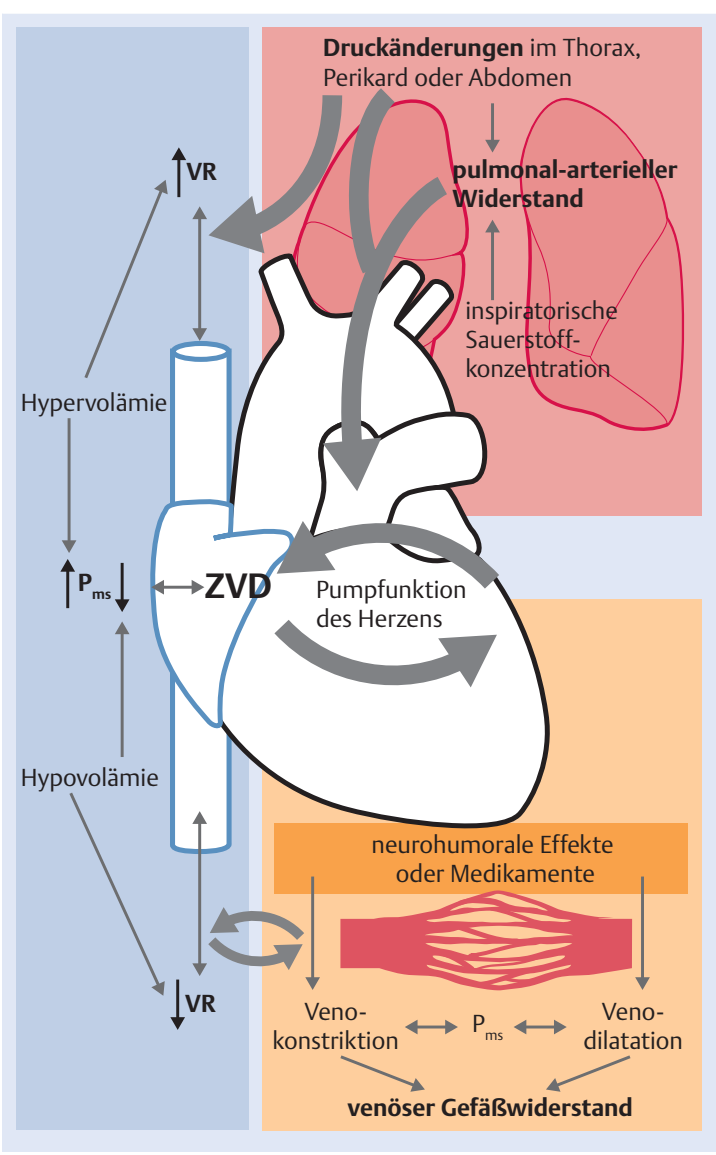

Volumensubstitution den ZVD steigen. Dabei ist die Änderung des ZVD in beide Richtungen nur solange erkennbar, bis eine kompensatorische Venodilatation oder Venokonstriktion einsetzt [40].

Ein normwertiger ZVD bedeutet keinesfalls, dass eine Normovolämie vorliegt.

Eine Mobilisierung von Blut durch Venokonstriktion u.a. aus venösen Kapazitätsgefäßen des Splanchnikusgebiets kann dazu führen, dass relevante Blutverluste ohne eine Reduktion des ZVD einhergehen [41]. Anderseits kann ein normwertiger ZVD auch bei einer kompensierten Hypervolämie auftreten, wenn Blut durch eine Vasodilatation im venösen Kapazitätssystem akkumuliert [35]. Des Weiteren kann bei orthostatisch bedingter Venodilatation ein deutlich erniedrigter ZVD trotz normalem Blutvolumen auftreten. Diese Beispiele verdeutlichen, welchen bedeutenden Einfluss der Venentonus auf den ZVD ausübt.

Herzfunktion. Auch die Funktion des Herzens als eine Druck-Saug-Pumpe beeinflusst den ZVD, wenn Blut während der Diastole infolge einer Verschiebung der Ventilebene in den rechten Ventrikel gesaugt wird
(Abb.1). Wie oben erläutert, führt die Pumpfunktion des Herzens darüber hinaus zum Anstieg des Drucks im arteriellen System, während im venösen System der Druck (also der ZVD) sinkt [40].

Zusammengefasst sind die multiplen Einflussfaktoren der Grund dafür, dass die exakte Ursache einer ZVDVeränderung im Einzelfall bettseitig oft nur schwer zu differenzieren ist. In Tab. 2 sind mögliche Ursachen für eine Steigerung oder Senkung des ZVD aufgeführt, Abb. 2 stellt die beeinflussenden Faktoren des ZVD grafisch dar.

\section{Der ZVD in der modernen Intensivmedizin: Immer noch ein treuer Freund oder längst überholt?}

Die Bedeutung des ZVD im intensivmedizinischen Alltag lässt sich in 4 Bereiche gliedern:

- Beurteilung des Volumenstatus

- Marker einer Rechtsherzbelastung oder Herzinsuffizienz

- Analyse der zentralen Venenpulskurve

- Indikator von Komplikationen und Prädiktor des Outcomes

\section{Evidenz zur Beurteilung des Volumenstatus}

Zweifelsohne ist der ZVD beim intensivmedizinischen Patienten ein relativ leicht zu erhebender Parameter und dementsprechend elegant erscheint die Steuerung der Flüssigkeitstherapie anhand des ZVD. Internationale Empfehlungen zur Volumentherapie - u.a. die Surviving Sepsis Campaign (SSC) - empfehlen dies bis heute bei schwerstkranken Patienten [42]. Was jedoch augenscheinlich eine direkte Stellgröße der kardialen Vorlast zu sein scheint, entpuppt sich bei genauer Betrachtung als Effekt multipler Einflussgrößen in einem komplexen physiologischen System.

Allgemein dient eine Flüssigkeitstherapie der Optimierung der Vorlast mit dem Ziel, das Herzzeitvolumen zu steigern. Die Vorlast ist dabei definiert als die enddiastolische Dehnung der myokardialen Sarkomere infolge der Ventrikelfüllung-zusammen mit der Nachlast und Kontraktionsfähigkeit des Herzens ist sie eine Determinate des Herzzeitvolumens [43]. 


\section{Tabelle 2}

Einflussfaktoren auf den zentralen Venendruck (mod. nach $[15,35]$ ).

\section{Gründe für eine Steigerung des ZVD}

Hypervolämie

Venokonstriktion

Steigerung des pulmonalarteriellen Widerstands

Druckerhöhung im Thorax oder Abdomen

\section{Gründe für eine Erniedrigung des ZVD}

ausgeprägte Hypovolämie
- z. B. Volumentherapie, Transfusion

- vegetative Regulation (Steigerung des Sympathikotonus)

- infolge von Medikamenteneffekten (z. B. Vasopressoren)

- Klappenvitien (z. B. Trikuspidalinsuffizienz)

- Herzinsuffizienz (sowohl rechtsbetont als auch global)

- Arrhythmien

- Myokardischämie

- Lungenembolie

- hypoxische Vasokonstriktion (Euler-Liljestrand-Mechanismus)

- kontrollierte Beatmung

- kontrollierte Beatmung (v. a. mit positivem endexspiratorischem Druck)

- forcierte Exspiration/Auto-PEEP

- Perikardtamponade

- Pneumothorax

- Spannungspneumothorax

- Trendelenburg-Lagerung

- Diabetes insipidus

- Blutverlust, hypovolämischer Schock

- Dehydratation

- starkes Erbrechen

- vegetative Regulation (z. B. Reduktion des Sympathikotonus)

- infolge von Medikamenteneffekte (z. B. Vasodilatatoren)

- u. a. Inotropika, Inodilatatoren, Diuretika

- forcierte Inspiration

- inspiratorischer Stridor
Druckabnahme im Thorax oder Abdomen (negativer Druck führt teils zum Kollaps der großen Venen) 
Füllungsdrücke und Füllungsvolumina. Die Füllungsdrücke des rechten Ventrikels, näherungsweise bestimmbar über den ZVD, korrelieren aufgrund der hohen Variabilität in der Compliance der Ventrikel jedoch nur unzureichend mit der enddiastolischen Füllung des Ventrikels oder dem Dehnungszustand des Myokards [3, 44]. Darüber hinaus wird der Druck im rechten Vorhof, also der ZVD, von einer Vielzahl an Faktoren beeinflusst. Dies bedeutet, anhand des ZVD als statischem Vorlastparameter kann man nicht abschätzen, auf welchem Niveau der Frank-StarlingKurve (Zusammenhang zwischen enddiastolischer Füllung und Auswurfleistung des Herzens) sich der Patient hämodynamisch befindet. Übrigens wäre dies auch durch eine exakte Punktschätzung der aktuellen Vorlast nicht möglich, da die Frank-Starling-Kurve für jeden Patienten individuell unterschiedlich sein kann. Abb. 4 zeigt schematisch das Verhalten von Herzzeitvolumen und ZVD: Gleiche Werte des ZVD können bei unterschiedlichem Herzzeitvolumen gemessen werden. Dem ZVD ist es als Momentaufnahme nicht möglich, eine Auskunft über diesen Zusammenhang zu geben.

Klinische Beobachtungen stützen diese Erkenntnis: Weder führen relevante Blutverluste zwingend zu einer Veränderung des ZVD [41] noch korreliert das Blutvolumen im Körper mit dem ZVD [4]. Kein Zusammenhang zwischen ZVD (bzw. dessen Änderung) und der Volumenreagibilität wurde gefunden [4]. Der Volumenstatus beim septischen Patienten korreliert ebenfalls nur unzureichend mit dem ZVD [45 - 47].

Beruhend auf dem Zusammenhang zwischen enddiastolischer Füllung und Auswurfleistung des Herzens scheinen messbare Füllungsvolumina den Füllungsdrücken bei der Beurteilung der Vorlast überlegen zu sein $[45,47,48]$. Beispielsweise korreliert das mit Thermodilution ermittelte globale enddiastolische Volumen (das Blutvolumen in allen 4 Herzhöhlen zum Zeitpunkt der Enddiastole) bei Patienten im septischen Schock besser mit dem Schlagvolumen als der ZVD [47]. Wie für die Füllungsdrücke gilt jedoch auch für die statischen Füllungsvolumina, dass auch sie nicht zufriedenstellend abschätzen lassen, auf welchem Niveau der Frank-Starling-Kurve der Ventrikel gerade pumpt $[49,50]$. So ist die Pumpfunktion des Herzens abhängig von der Vorlast - unklar ist allerdings, bei welcher Vorlast der Ventrikel „optimal“ gefüllt ist. Offensichtlich sind dynamische Parameter der Volumenreagibilität (z.B. Schlagvolumenvariation) hier den statischen Parametern überlegen [51].
„Early goal-directed therapy“. Die 2012 von der SSC veröffentlichten Leitlinien zur Therapie der Sepsis empfehlen auch heute noch den Gebrauch des ZVD als Zielparameter einer Volumentherapie bei schwerer Sepsis und septischem Schock [42]. Die Empfehlung ist einer randomisierten, kontrollierten Single-CenterStudie entnommen, die 2001 von Rivers et al. vorgestellt wurde. Das Studienprotokoll dieser „early goaldirected therapy“, welches in der Interventionsgruppe einen ZVD von 8-12 mmHg, einen mittleren arteriellen Druck von 65-90 mmHg und eine zentralvenöse Sauerstoffsättigung von mindestens $70 \%$ anstrebte, führte zu einer absoluten Reduktion der 28-Tage-Mortalität von 15,9\% [52].

Die aus der „early goal-directed therapy“ in die Empfehlungen der SSC übernommenen Therapieziele sind jedoch nicht unumstritten. Jüngst konnten 2 randomisierte, multizentrische Studien (ARISE und ProCESS) den positiven Effekt bei Patienten im septischen Schock nicht bestätigen [53,54]. Im Vergleich zur Rivers-Studie ähnelten sich die Flüssigkeitstherapien im Studien- und Kontrollarm von ARISE und ProCESS jedoch. Insgesamt war die Flüssigkeitstherapie restriktiver und der Einsatz von Vasopressoren frühzeitiger, was mutmaßlich zu einem - im Vergleich zur Rivers-Studie - verbesserten Outcome in allen Therapiegruppen beigetragen hat [55].

Bei Patienten mit septischem Schock erreichte eine randomisierte klinische Studie die geringste Mortalität bei Patienten mit den niedrigsten ZVD-Werten (unter $8 \mathrm{mmHg}$ ). Die höchste Sterblichkeit hatten Patienten mit einem ZVD von über $12 \mathrm{mmHg}$ [56].

Eine am ZVD orientierte Volumentherapie ist bei der Sepsis oft mit einer Flüssigkeitsakkumulation verbunden. Dies ist ein hochsignifikanter Prädiktor der Mortalität und Organschädigung beim kritisch Kranken [56].

Akute Nierenschädigung. Legrand et al. fanden eine lineare Korrelation zwischen der Höhe des ZVD und der Wahrscheinlichkeit eines akuten Nierenversagens bei Patienten mit schwerer Sepsis. Die Assoziation blieb auch nach multivariater Adjustierung signifikant $(\mathrm{OR}=1,22$ [1,08-1,39] pro $1 \mathrm{mmHg} Z \mathrm{ZVD}, \mathrm{p}=0,002)$. Im Bereich 8-12 mmHg lag das Risiko einer akuten Nierenschädigung bei ca. $50 \%$, fiel bei Werten darunter steil ab und stieg bei Werten darüber steil an [79]. Mit diesem Wissen erscheint es fragwürdig, bei kritisch kranken Patienten per Protokoll einen hohen Wert für eine Variable aktiv anzustreben, die bei hohen Werten mit Komplikationen assoziiert ist. 
In der deutschen S3-Leitlinie zur Volumentherapie wird von der Verwendung des ZVD zur Steuerung einer Volumentherapie abgeraten [57].

ZVD zur Beurteilung des Volumenstatus. Welche Rückschlüsse können aus dem ZVD zur Beurteilung des Volumenstatus gezogen werden? Stéphan et al. versuchten anhand eines klinischen Scoring-Systems die zirkulierende Blutmenge bei Intensivpatienten zu beurteilen [58]. Zum Vergleich wurde die zirkulierende Blutmenge durch Injektion von mit ${ }^{125}$ I radioaktiv markiertem Humanalbumin bestimmt. Keines der untersuchten klinischen Zeichen und keine der hämodynamischen Variablen hatte einzeln betrachtet einen klinisch nützlichen prädiktiven Wert zur Beurteilung der zirkulierenden Blutmenge. Ein komplexes, multivariates Scoring-System war in dem untersuchten Patientenkollektiv nützlich, die zirkulierende Blutmenge einzuschätzen. Aus den Studienergebnissen folgerten die Autoren im Hinblick auf den ZVD, dass dieser allenfalls bei extremen Werten eine klinische Relevanz zur Beurteilung der zirkulierenden Blutmenge hat [58]. Es bleibt also für die klinische Praxis, dass beispielsweise der klinische Verdacht auf eine dekompensierte Hypovolämie durch einen extrem niedrigen ZVD gestützt wird, während der Verdacht auf eine Perikardtamponade durch sehr hohe ZVD-Werte erhärtet wird. Im häufigen intermediären Bereich hilft uns der ZVD hier nicht.

\section{Analyse der zentralen Venenpulskurve}

Durch eine kontinuierliche Bestimmung des ZVD über die Zeit können auf einem Monitor die vom Herzzyklus abhängigen Veränderungen der zentralen Venenpulskurve aufgezeichnet werden. Der Füllungsdruck des rechten Ventrikels wird vereinfacht aus dem zeitlichen Mittelwert der zentralen Venenpulskurve bestimmt. Charakteristische Veränderungen der zentralen Venenpulskurve lassen diagnostische Rückschlüsse zu. Eine Auswahl pathologischer Veränderungen der zentralen Venenpulskurve zeigt Abb.3. Schwierigkeiten bei der Interpretation der Venenpulskurve bereitet u.a. eine erhöhte Herzfrequenz (Tachykardie), da dann die einzelnen Wellen und Senken nur schwer zu unterscheiden sind [27].

Das gleichzeitige Ableiten eines EKG erleichtert das Identifizieren der Wellen und Senken der zentralen Venendruckkurve (Abb.3). Physiologischerweise ist die a-Welle größer oder gleich groß wie die v-Welle. Liegt eine ausgeprägte Trikuspidalinsuffizienz vor, tritt eine

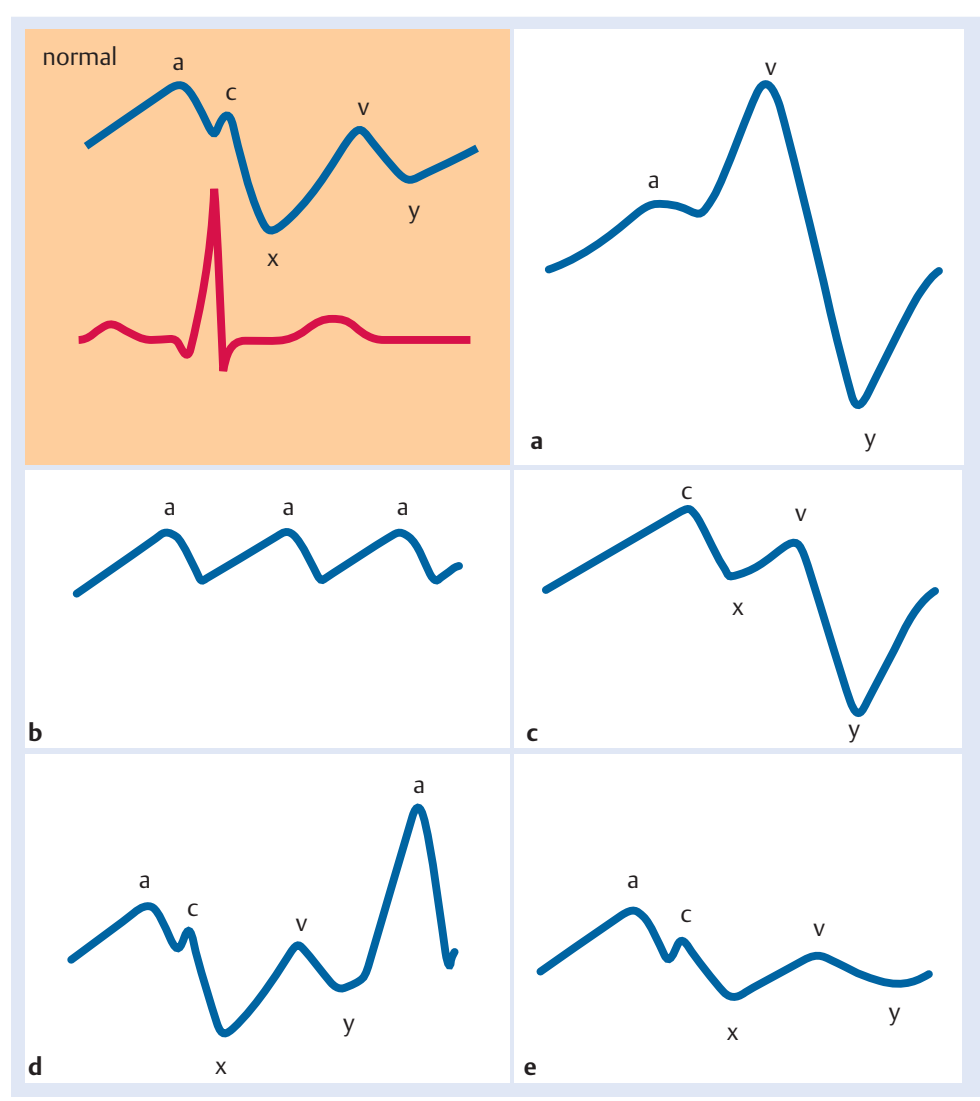

Abb. 3 Pathologische Veränderungen der zentralen Venenpulskurve [27,65]. a Trikuspidalinsuffizienz: Verlust der c-Welle durch Regurgitation von Blut in den rechten Vorhof während der Ventrikelkontraktion. b Vorhofflattern: repetitive a-Wellen durch Kontraktion des Vorhofs. c Vorhofflimmern: Verlust der a-Welle durch funktionellen Stillstand des rechten Vorhofs. d AV-Block III': prominente a-Welle bei Kontraktion des rechten Vorhofs gegen die geschlossene Trikuspidalklappe (Propfungswelle). Stark ausgeprägte a-Wellen finden sich außerdem bei AV-Knotenrhythmus, AV-Dissoziation, unter Katecholamintherapie und bei ventrikulärer Schrittmacherstimulation. e Perikardtamponade: Reduktion der passiven Vorhoffüllung (x-Senke und y-Senke) durch Erhöhung des intraperikardialen Drucks.

prominente v-Welle auf, die eine deutlich größere Amplitude als die a-Welle hat und deren Ursache die Regurgitation von Blut in den rechten Vorhof ist [65]. Bei Patienten mit einer relevanten Insuffizienz der Trikuspidalklappe liegt häufig auch eine Funktionseinschränkung der Niere mit reduzierter glomerulärer Filtrationsrate vor, womöglich aufgrund der venösen Regurgitation mit Blutrückstau in die Nieren (s. o.) [66].

Typischerweise zeigt eine Perikardtamponade einen Verlust der $\mathrm{x}$ - und $\mathrm{y}$-Senke in der zentralen Venenpulskurve (Abb.3e). Ursache für den Verlust beider Senken ist der Druckanstieg im Perikard. Bei konstantem intraperikardialem Druck kann Blut nur während der Systole (Kontraktion des Ventrikels) in den rechten Vorhof strömen. Dieses Blut strömt während der Diastole durch die geöffnete Trikuspidalklappe, dabei bleibt der intraperikardiale Druck konstant. Eine 
passive Füllung des rechten Vorhofs durch Reduktion des intraperikardialen Drucks ( $\mathrm{x}$ - und $\mathrm{y}$-Senke) tritt nicht auf [65].

Wird bei einem Patienten der ZVD bestimmt, sollte man auch die zentrale Venenpulskurve begutachten, da diese wertvolle Informationen liefern kann.

\section{Der ZVD als Marker einer Rechtsherz- belastung oder Herzinsuffizienz}

Bei der (chronischen) Rechtsherzinsuffizienz sind infolge einer Volumenretention und Steigerung des Venentonus die Drücke in den zentralen Venen erhöht. Die Vorstellung einer venösen Stauung vor dem Herzen („Stauungsinsuffizienz“) als Ursache des erhöhten ZVD trifft insofern nicht zu, als dass vielmehr eine Verschiebung des Gleichgewichts von Blutvolumen, Kapazität des Gefäßsystems und Herzleistung besteht [28]: Um dies zu zeigen, maß Starr den statischen Blutdruck (s.o.) von frischen Leichen bei zu Lebzeiten Kreislaufgesunden und bei Patienten, die infolge eines chronischen Herzinsuffizienz mit erhöhten Venendrücken verstorben waren. Bei Ersteren betrug der statische Blutdruck 7,6 $\mathrm{cmH}_{2} \mathrm{O}$ (ca. $5 \mathrm{mmHg}$ ), bei den Patienten, die infolge der chronischen Herzinsuffizienz gestorben waren, $\geq 20 \mathrm{cmH}_{2} \mathrm{O}$ (ca. $15 \mathrm{mmHg}$ ) [28].

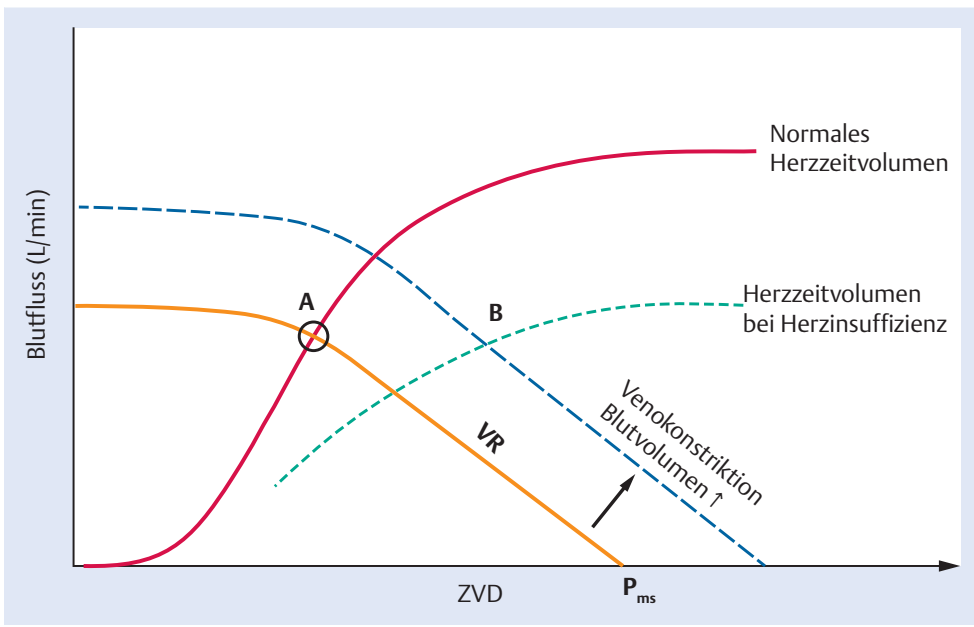

Abb. 4 Analyse des Kreislaufs nach Guyton (bei konstanter Herzfrequenz und konstantem arteriellem Druck) [40]. In einem Koordinatensystem ist der zentrale Venendruck (ZVD) gegenüber dem Blutfluss aufgetragen. Im geschlossenen Kreislauf müssen sich Herzzeitvolumen (HZV) und venöser Rückstrom (VR) zum Herzen entsprechen.

Der Schnittpunkt A der Kurve des HZV mit der des VR markiert diese Situation im gesunden Kreislauf. Bei der Herzinsuffizienz (gepunktete Kurve) ist die Kurve des HZV abgeflacht, kompensatorisch sind Blutvolumen und Venentonus erhöht. Die Kurve des VR ist nach oben verschoben.

Der Punkt B entspricht dem neuen Fließgleichgewicht des Kreislaufs mit einem ähnlichen $H Z V$, aber deutlich gesteigertem ZVD. Der Schnittpunkt der VR-Kurve mit der X-Achse $(\mathrm{HZV}=0 \mathrm{l} / \mathrm{min})$ entspricht dem mittleren Füllungsdruck des Kreislaufs $\left(P_{\mathrm{ms}}\right)$.
Blutvolumen und Füllungsdruck. Die Zunahme des Blutvolumens und der Anstieg des Füllungsdrucks sind als Ausdruck eines Kompensationsmechanismus des Herzens zur Aufrechterhaltung eines ausreichenden Herzzeitvolumens aufzufassen. Um seine Kontraktionsfähigkeit gemäß dem Frank-Starling-Mechanismus aufrechtzuerhalten, benötigt der beeinträchtigte rechte Ventrikel einen höheren Füllungsdruck und ein größeres enddiastolisches Volumen (Abb.4). Dem Anstieg des Blutvolumens liegt pathophysiologisch u. a. eine Reduktion des renalen Blutflusses zugrunde, wodurch das Renin-Angiotensin-Aldosteron-System aktiviert wird. Dies führt zur vermehrten Retention von Natrium und Wasser und damit zur Steigerung des intravasalen Volumens. Zum Anstieg des ZVD führt u.a. der gesteigerte Sympathikotonus mit erhöhten Konzentrationen zirkulierender Katecholamine. Dies führt im venösen System v.a. zur Venokonstriktion in kutanen Venen und im Splanchnikusgebiet [40]. Umgekehrt führt die erhöhte Vorlast - sowohl bei Herzinsuffizienz als auch infolge einer Volumentherapie - zur Aktivierung des natriuretischen Peptid-Systems, sodass vermehrt Flüssigkeit ausgeschieden wird. Damit sinkt der systemvaskuläre Widerstand durch Vasodilatation und durch die Steigerung der Kapillarpermeabilität entstehen Ödeme [59, 60]. Diese komplexe Regulation betont übrigens ebenfalls, warum eine Steuerung der Volumentherapie anhand des ZVD deletär sein kann. Schließlich führt jede Volumenbelastung mit Dehnung der Herzvorhöfe zur Initiation der HerzinsuffizienzKaskade mit Steigerung der Diurese, arterieller Vasodilatation und Kapillarleck.

Rechtsherzinsuffizienz. Ursache für die Entwicklung einer Rechtsherzinsuffizienz kann primär eine kardiale Ursache sein (z.B. Myokardinfarkt, Arrhythmien oder Klappenvitien). Eine Rechtsherzinsuffizienz kann auch sekundär durch extrakardiale Umstände hervorgerufen werden (z.B. Lungenembolie, Perikardtamponade; Tab. 2). In Verbindung mit einem erhöhten ZVD - meist bei chronischem Verlauf - kann man oft schon während der klinischen Untersuchung Zeichen einer Rechtsherzinsuffizienz nachweisen. Dies sind typischerweise Gewichtszunahme, periphere Ödeme oder Aszites [61-63].

Allein aus einem erhöhten ZVD sollte man nicht unkritisch auf das Vorliegen oder die Progredienz einer Rechtsherzinsuffizienz schließen. 
Linksherzinsuffizienz. Auch eine Linksherzinsuffizienz kann einen Anstieg des ZVD verursachen. Die erhöhten linksatrialen Drücke werden über die Lungenstrombahn zum rechten Herzen weitergeleitet und steigern den Druck im rechten Vorhof bzw. den ZVD. Ein erhöhter Druck in der Jugularvene als Surrogatmarker eines erhöhten ZVD ist bei Patienten mit Linksherzinsuffizienz ein ungünstiger prognostischer Marker, u.a. bezüglich der Progression der Erkrankung und dem Tod infolge eines Pumpversagens (relatives Risiko 1,37; 95\%-Konfidenzintervall 1,07-1,75; p<0,01) [64]. Hier ist der ZVD somit v. a. ein prognostischer Marker. Des Weiteren wird er für die Berechnung zahlreicher hämodynamischer Variablen zur Beurteilung der Rechtsherzinsuffizienz verwendet.

\section{Der ZVD als Indikator von Komplikationen und Prädiktor des Outcomes}

Kardiorenales Syndrom. Patienten mit Herzinsuffizienz entwickeln in bis zu 50\% der Fälle eine Verschlechterung der Nierenfunktion. Dieser Symptomkomplex wird als kardiorenales Syndrom bezeichnet [69-71]. Eine Verschlechterung der Nierenfunktion ist ein Prädiktor eines ungünstigen Verlaufs und einer gesteigerten Letalität in dieser Patientengruppe $[72,73]$. Traditionell besteht die Annahme, dass sich eine Verschlechterung der Nierenfunktion infolge eines verminderten Herzzeitvolumens mit einem reduzierten renalen Blutfluss entwickelt [74]. Darüber hinaus lassen aktuelle Studien jedoch vermuten, dass v.a. die Zunahme des Blutvolumens und der erhöhte rechtsventrikuläre Füllungsdruck (d.h. ZVD) pathophysiologisch entscheidend zur Verschlechterung der Nierenfunktion beitragen $[75,76]$.

Vermutlich ist dieser Effekt auf einen Druckanstieg im efferenten Ende der glomerulären Kapillaren, welcher den Filtrationsdruck herabsetzt, zurückzuführen [77]. Mullens et al. untersuchten zur Prüfung dieser Hypothese 145 intensivmedizinische Patienten mit fortgeschrittener dekompensierter Herzinsuffizienz (linksventrikuläre Ejektionsfraktion<30\%) [75]. Eine Verschlechterung der Nierenfunktion wurde bei einem Anstieg des Serumkreatinins um $\geq 0,3 \mathrm{mg} / \mathrm{dl}$ angenommen. Patienten mit einem höheren ZVD bei Aufnahme auf die Intensivstation (ZVD $18 \pm 7$ vs. $12 \pm 6 \mathrm{mmHg}$, $\mathrm{p}<0,001)$ und unter intensivmedizinischer Therapie ( $11 \pm 8$ vs. $8 \pm 5 \mathrm{mmHg}, \mathrm{p}=0,04$ ) entwickelten häufiger eine Verschlechterung der Nierenfunktion als Patienten mit einem ZVD unter $8 \mathrm{mmHg}(\mathrm{p}=0,01)$. Der Herzindex war bei Aufnahme signifikant höher bei den
Patienten, die eine Verschlechterung der Nierenfunktion entwickelten $\left(2,0 \pm 0,8\right.$ vs. $1,8 \pm 0,41 / \mathrm{min} / \mathrm{m}^{2}$, $\mathrm{p}=0,008)$. Von den Patienten in der Studiengruppe mit einem ZVD von über $24 \mathrm{mmHg}$ entwickelten $75 \%$ eine Verschlechterung der Nierenfunktion. Aus ihrer Studie folgerten Mullens et al., dass die Volumenbelastung des venösen Systems mit gesteigertem ZVD bei der fortgeschrittenen dekompensierten Herzinsuffizienz der wichtigste hämodynamische Faktor einer Verschlechterung der Nierenfunktion sei [75].

Die Gruppe um Iacoviello fand bei 245 Patienten, bei denen die Diagnose einer chronischen Herzinsuffizienz (linksventrikuläre Ejektionsfraktion $\leq 45 \%$ ) nach Kriterien der European Society of Cardiology gestellt wurde, dass ein erhöhter ZVD (definiert als $>5 \mathrm{mmHg}$ ) eine unabhängige Variable bei der Entwicklung einer Verschlechterung der Nierenfunktion ist (OR 4,68, 95\%Konfidenzintervall 2,15-10,21, p=0,001) [76].

Im Gegensatz dazu fanden Uthoff et al. bei 140 Patienten, die mit Symptomen einer akuten Herzinsuffizienz hospitalisiert wurden, dass Patienten mit niedrigerem ZVD ( $<10 \mathrm{cmH}_{2} \mathrm{O}$, ca. $<7,4 \mathrm{mmHg}$ ) bei Aufnahme häufiger in den ersten 24 Stunden eine Verschlechterung der Nierenfunktion entwickelten als Patienten mit einem höheren ZVD (> $15 \mathrm{cmH}_{2} \mathrm{O}$, ca. > $11 \mathrm{mmHg}$; 18\% vs. $4 \%, p=0,046)$. Andererseits hatten Patienten, die gleichzeitig einen geringen systolischen Blutdruck (<110 mmHg) und einen höheren ZVD (> $15 \mathrm{cmH}_{2} \mathrm{O}$, ca. $>11 \mathrm{mmHg}$ ) aufwiesen, eine signifikant niedrigere glomeruläre Filtrationsrate [61].

Bei genauer Betrachtung können die 3 Studien nicht uneingeschränkt miteinander verglichen werden, da sie u.a. unterschiedliche Patientenkollektive einschlossen (dekompensierte chronische vs. akute Herzinsuffizienz). Insgesamt mehren sich jedoch die Hinweise darauf, dass die Störung der Nierenfunktion im kardiorenalen Syndrom wesentlich durch die venöse Kongestion mit ZVD-Erhöhung mitbedingt ist $[71,78]$. Bei septischen Patienten ist die Höhe des ZVD ebenfalls mit der Entwicklung eines akuten Nierenversagens assoziiert. Vermutlich wird das Nierenversagen bei septischen Patienten ebenfalls durch den venösen Rückstau von Blut in die Nieren beeinflusst [79]. Die Empfehlung der SSC, einen als physiologisch bezeichneten ZVD (8$12 \mathrm{mmHg}$ ) als Zielparameter anzustreben, ist vor diesem Hintergrund als paradox zu betrachten $[2,42]$.

Die Autoren um Williams fanden bei 2390 HochrisikoPatienten, die sich einer koronaren Bypassoperation unterzogen, einen signifikanten Zusammenhang zwi- 
schen der Höhe des ZVD und der 30-Tage- bzw. Krankenhausletalität sowie dem Auftreten eines Nierenversagens [80]. Die Ergebnisse waren unabhängig vom Herzindex sowie von anderen klinischen Variablen. Für jeden Anstieg des ZVD um 5 mmHg 6 Stunden nach Aufnahme auf die Intensivstation stieg die risikoadjustierte OR für die Entwicklung eines Nierenversagens oder den Tod innerhalb von 30 Tagen bzw. im Krankenhaus um 1,54 (95\%-Konfidenzintervall 1,28-1,86; $\mathrm{p}<0,0001)$. Für die Entwicklung eines Nierenversagens allein betrug die OR pro $5 \mathrm{mmHg}$ Anstieg des ZVD 6 Stunden nach der Aufnahme auf die Intensivstation 5,5 (95\%-Konfidenzintervall 1,93-15,5; p=0,001) bezogen auf Patienten mit einem ZVD unter $9 \mathrm{mmHg}$. Für Patienten mit einem ZVD ab $9 \mathrm{mmHg}$ betrug die risikoadjustierte OR 1,3 (95\%-Konfidenzintervall $1,01-1,65 ; p=0,045$ ) [80]. Die Autoren der Studie folgerten, dass womöglich in Zukunft die Prognose der Patienten durch eine Therapiesteuerung anhand des postoperativen ZVD verbessert werden könnte [80] allerdings nicht durch eine gezielte Steigerung, sondern durch eine Reduktion des ZVD.

Rady et al. zeigten ebenfalls den prädiktiven Charakter des ZVD bei kardiochirurgischen älteren Patienten. Sowohl eine präoperative Erhöhung des ZVD auf über $15 \mathrm{mmHg}$ oder eine Erhöhung des ZVD bei Aufnahme auf die Intensivstation waren mit einer signifikant höheren Morbidität und Mortalität assoziiert [81].

Komplikationen nach Lungentransplantation. Nicht nur bei kardiochirurgischen Patienten, auch bei Patienten nach einer Lungentransplantation ist ein erhöhter ZVD (>7 mmHg) mit dem Auftreten von Komplikationen assoziiert (verlängerte mechanische Beatmung, erhöhte Mortalität auf der Intensivstation und erhöhte Krankenhausmortalität) [82].

Komplikationen nach Lebertransplantation. Im Rahmen der Leberchirurgie scheint der ZVD mit einer erhöhten Rate von Blutungen und Leberfunktionsstörungen einherzugehen. Ein niedriger ZVD führt bei Leberresektionen zu kürzeren Operationszeiten, weniger Blutverlust und geringeren Transfusionsraten [83]. Interessanterweise kommt es nach Leberoperationen unter Aufrechterhaltung eines niedrigen ZVD nicht zu postoperativen Störungen der Nierenfunktion [83,84]. Eine geringere Komplikationsrate bei niedrigem ZVD wurde auch für den perioperativen Verlauf von Lebertransplantationen nachgewiesen [85].
Zusammengefasst scheinen pathologische Werte des ZVD, aber auch Werte im oberen „physiologischen“ Bereich in bestimmten Patientenkollektiven ein wichtiger Prädiktor für die Entwicklung von Komplikationen zu sein. Erhöhte Werte bei kardiovaskulären Erkrankungen sind assoziiert mit einer beeinträchtigten Nierenfunktion, der Wiederaufnahme ins Krankenhaus und darüber hinaus mit einer gesteigerten Mortalität [61, 78, 86].

Bislang ist nicht hinreichend untersucht worden, ob eine aktive Senkung des ZVD zur Vermeidung von Komplikationen beitragen kann. Ist bei einem intensivmedizinischen Patienten ein ZVK gelegt worden, kann die Messung des ZVD das Gesamtbild vervollständigen und eventuell um eine Aussage zur Prognose ergänzen.

\section{Schlussfolgerung}

Die Tatsache, dass im klinischen Alltag häufig Drücke anstelle von Flüssen und Volumina gemessen werden, liegt an der einfacheren technischen Verfügbarkeit einer Druckmessung. Um aus dem ZVD diagnostische Schlüsse ziehen zu können, sind einige Dinge zu beachten:

- technisch korrekte Messung

- Berücksichtigung intrathorakaler Druckverhältnisse

- Berücksichtigung dynamischer Faktoren (u.a. Venentonus)

Als alleiniger Parameter ist der ZVD nicht geeignet, Aussagen über den Volumenstatus oder die Volumenreagibilität zu treffen. Hier scheinen moderne, dynamische Parameter dem ZVD überlegen zu sein. Die Steuerung einer Volumentherapie einzig am ZVD ist obsolet und wahrscheinlich schädlich, da eine mögliche venöse Volumenüberladung zu Organfunktionsstörungen beitragen kann.

Dass der ZVD allerdings aufgrund seiner fehlenden Eignung als Vorlastparameter selbst unter Druck geraten ist, wird seiner pathophysiologischen Bedeutung nicht vollständig gerecht: Der ZVD und die zentrale Venenpulskurve sind weiterhin relevante diagnostische Werkzeuge, u.a. bei der Diagnostik einer Perikardtamponade. Hier gilt, dass insbesondere extrem hohe oder niedrige ZVD-Werte eine diagnostische Relevanz haben, während intermediäre Werte selten hilfreich sind. Darüber hinaus ist der ZVD bei bestimmten Patientengruppen ein Prädiktor für die Mortalität und das Auftreten von Komplikationen. So 


\section{Kernaussagen}

- Um aus dem ZVD therapeutische Schlüsse ziehen zu können, sind einige Dinge zu beachten: (1) technisch korrekte Messung, (2) Berücksichtigung intrathorakaler Druckverhältnisse und (3) Berücksichtigung dynamischer Faktoren (u.a. Venentonus). Die Bewertung des ZVD, v. a. bei plötzlichen Änderungen, muss zwingend unter Kenntnis möglicher Fehlerquellen und im klinischen Kontext des Patienten erfolgen.

- Die zentrale Venenpulskurve weist einen typischen Verlauf, gekennzeichnet durch Wellen und Senken, auf. Pathologien können sich in charakteristischen Veränderungen des Kurvenverlaufs zei- gen. Daher können aus der Analyse der zentralen Venenpulskurve wertvolle Informationen über den Patienten gewonnen werden.

- Als alleiniger Parameter ist der zentrale Venendruck nicht geeignet, Aussagen über den Volumenstatus und die Volumenreagibilität zu treffen. Des Weiteren ist die Steuerung einer Volumentherapie einzig am ZVD obsolet und wahrscheinlich schädlich, da eine mögliche venöse Kongestion zu Organfunktionsstörungen beitragen kann.

- In bestimmten Patientenkollektiven (u. a. Sepsis und Herzinsuffizienz) ist der zentrale Venendruck ein relevanter
Indikator für die Entwicklung von Komplikationen und ein Prädiktor des Outcomes.

- Die aktive Senkung des ZVD zur Vermeidung der mit venöser Kongestion assoziierten Komplikationen ist Gegenstand aktueller Forschung.

- Da der ZVD beim intensivmedizinischen Patienten insgesamt v. a. ein prognostischer Marker ist und bisher keine ausreichende Evidenz zur Therapiesteuerung anhand des ZVD vorliegt, sollte auf die Anlage eines ZVK zur alleinigen Messung des ZVD verzichtet werden. gehen erhöhte ZVD-Werte bei septischen und herzinsuffizienten Patienten mit einer erhöhten Sterblichkeit und einem häufigeren Nierenversagen einher. Zukünftige Studien werden zeigen, ob die aktive Senkung des ZVD („weaning the CVP down“) bei bestimmten Patientengruppen zu einer Verbesserung des Outcomes beitragen kann.
Da der ZVD beim intensivmedizinischen Patienten insgesamt v.a. ein prognostischer Marker ist und bisher keine ausreichende Evidenz zur Therapiesteuerung anhand des ZVD vorliegt, sollte man auf das Anlegen eines ZVK zur alleinigen Messung des ZVD verzichten.

Interessenkonflikt: Die Autoren geben an, dass keine Interessenkonflikte bestehen. 


\section{Über die Autoren}

\section{Michael Heßler}

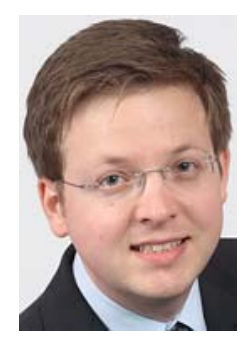

Jahrgang 1987. 2007-2013 Studium der Humanmedizin an der Westfälischen Wilhelms-Universität Münster. Seit 2009 Mitarbeit in der Arbeitsgruppe „Sepsis und Multiorganversagen" an der Klinik für Anästhesiologie, operative Intensivmedizin und Schmerztherapie des Universitätsklinikums Münster. Seit 2014 Arzt in

Weiterbildung im Fach Anästhesiologie und wissenschaftlicher Mitarbeiter an der Klinik für Anästhesiologie, operative Intensivmedizin und Schmerztherapie des Universitätsklinikums Münster.

\section{Philip-Helge Arnemann}

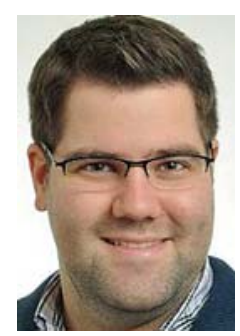

Dr. med. Jahrgang 1986. 2007-2013 Studium der Humanmedizin an der Westfälischen Wilhelms-Universität Münster. Seit 2009 Mitwirkung in der Arbeitsgruppe „Sepsis und Multiorganversagen" an der Klinik für Anästhesiologie, operative Intensivmedizin und Schmerztherapie des Universitätsklinikums Münster. Seit 2013 wissenschaftlicher Mitarbeiter und Assistenzarzt im Fach Anästhesiologie an der Klinik für Anästhesiologie, operative Intensivmedizin und Schmerztherapie des Universitätsklinikums Münster.

\section{Christian Ertmer}

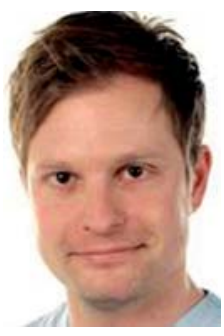

Priv.-Doz. Dr. med. Jahrgang 1979. 1999-2005 Studium der Humanmedizin an der Westfälischen WilhelmsUniversität Münster. Seit 2002 Mitarbeit in der Arbeitsgruppe „Sepsis und Multiorganversagen “ an der Klinik für Anästhesiologie, operative Intensivmedizin und Schmerztherapie des Universitätsklinikums Münster.

2006-2011 Facharztausbildung als Anästhesist und wissenschaftlicher Mitarbeiter in der Klinik für Anästhesiologie, operative Intensivmedizin und Schmerztherapie des Universitätsklinikum Münster. 2012 Venia legendi für das Fach Anästhesiologie und operative Intensivmedizin. Seit 2013 Oberarzt in der Klinik für Anästhesiologie, operative Intensivmedizin und Schmerztherapie des Universitätsklinikums Münster; Stellvertretender Leiter operative Intensivmedizin.

\section{Korrespondenzadresse}

Priv.-Doz. Dr. med. Christian Ertmer

Universitätsklinikum Münster

Klinik für Anästhesiologie, operative Intensivmedizin und Schmerztherapie

Albert-Schweitzer-Campus 1, Gebäude A1

48149 Münster

E-Mail: ertmer@anit.uni-muenster.de

Die Literatur zu diesem Beitrag finden Sie unter http://dx.doi.org/10.1055/s-0034-1392645. 


\section{CME-Fragen}

\section{CME.thieme.de}

\section{CME-Teilnahme}

- Viel Erfolg bei Ihrer CME-Teilnahme unter http://cme.thieme.de

- Diese Fortbildungseinheit ist 12 Monate online für eine CME-Teilnahme verfügbar.

- Sollten Sie Fragen zur Online-Teilnahme haben, unter http://cme.thieme.de/hilfe finden Sie eine ausführliche Anleitung.

\section{1}

Welche Aussage zur Messung des ZVD ist falsch?
A Die Messung des ZVD führte erstmals Werner Forssmann im Jahr 1905 durch.

B Der ZVD kann u. a. mit dem Prinzip der kommunizierenden Röhren bestimmt werden.

C Der hydrostatische Nullpunkt, auf den sich die Messung des ZVD bezieht, befindet sich ungefähr auf Höhe von vier Fünfteln des Thoraxdurchmessers, gemessen von der Rückseite des Patienten in Rückenlage.

D Bei Veränderung der Patientenposition muss die Position des Drucksensors angepasst werden.

E Die Position des Drucksensors wird in der Klinik häufig falsch gewählt.

\section{2}

Zu welchem Zeitpunkt sollte man unter Beatmung den ZVD am besten messen?

\section{3}

In der Literatur werden Normbereiche für den zentralen Venendruck angegeben. Welche Aussage zur Angabe des ZVD und dessen Umrechnung in andere Einheiten ist richtig?

4

Welche Reihenfolge von Wellen und Senken kennzeichnet typischerweise die zentrale Venenpulskurve beim gesunden Patienten?

5

Verschiedene Ursachen können zu einer Steigerung des ZVD führen. Was führt in der Regel nicht zu einem Anstieg des ZVD?
A endexspiratorisch während der Systole
B endexspiratorisch während der Diastole
C exspiratorisch
D inspiratorisch
E während des höchsten Beatmungsdrucks

A primäre Störung der kardialen Pumpfunktion

B Steigerung des pulmonalarteriellen Widerstands

C Hypervolämie

D Diabetes insipidus

E Druckerhöhung im Thorax oder Abdomen 


\section{CME-Fragen}

Under pressure - Der Stellenwert des zentralen Venendrucks in der modernen Intensivmedizin

6

Welche Aussage über die Evidenz zur Beurteilung des Volumenstatus anhand des ZVD ist richtig?

A Die deutsche S3-Leitlinie zur Volumentherapie rät von der Nutzung des ZVD zur Steuerung einer Volumentherapie ab.

B Der ZVD korreliert mit dem zirkulierenden Blutvolumen.

C Die Änderung des zentralen Venendrucks korreliert mit der Volumenreagibilität.

D Anhand des ZVD lässt sich abschätzen, auf welchem Niveau der Frank-Starling-Kurve (Zusammenhang zwischen Auswurfleistung und venösem Rückstrom zum Herzen) sich der Patient hämodynamisch befindet.

E Die Surviving Sepsis Campaign empfiehlt die Volumentherapie bei schwerstkranken Patienten nicht anhand des ZVD zu steuern.

\section{7}

Bei welcher Erkrankung tritt typischerweise eine Propfungswelle in der zentralen Venenpulskurve auf?

\section{8}

Der ZVD und die zentrale Venenpulskurve können als Indikatoren von Komplikationen und Prädiktoren des Outcomes herangezogen werden. Welche Aussage dazu ist falsch?
A hämodynamisch relevante Trikuspidalinsuffizienz
B Vorhofflattern
C AV-Block III
D Vorhofflimmern
E Perikardtamponade

A Entlastungswürdige Perikardtamponaden sind nach herzchirurgischen Eingriffen häufig (1-2\%) und sind typischerweise an einem Verlust der $\mathrm{x}$ - und $\mathrm{y}$-Senken in der zentralen Venenpulskurve zu erkennen.

B Nach einer koronaren Bypassoperation korreliert die Höhe des ZVD mit der 30-Tage- bzw. Krankenhausletalität sowie dem Auftreten eines Nierenversagens.

C Nach einer Lungentransplantation ist ein erhöhter ZVD $(>7 \mathrm{mmHg})$ mit dem Auftreten von Komplikationen assoziiert.

D Eine Korrelation zwischen der Höhe des ZVD und der Wahrscheinlichkeit eines akuten Nierenversagens wurde bei Patienten mit schwerer Sepsis nachgewiesen.

E Eine aktive Senkung des ZVD („weaning the CVP down“) ist ein evidenzbasiertes, weitläufig etabliertes Verfahren zur Verbesserung des Outcomes.

\section{9}

Die Beschreibung der Physiologie des ZVD ist wesentlich geprägt von den Theorien von Gauer und Guyton. Welche Aussage zu diesen Theorien ist richtig?
A Das Modell von Guyton basiert genau wie das Kreislaufmodell Starlings auf einem kardiozentrischem Kreislaufmodell.

B Der Zusammenhang der Determinanten des venösen Rückstroms (mittlerer Füllungsdruck des Blutkreislaufs, ZVD und Gefäßwiderstand) kann in Analogie zum Ohm-Gesetz dargestellt werden.

C Das Modell von Gauer basiert auf der Vorstellung des Kreislaufs als einem System unverbundener starrer Röhren.

D Guyton konzentriert sich in seinem Modell auf den arteriellen Abfluss vom Herzen.

E Gauer zeigte in Untersuchungen zu seinem Modell, dass die Volumenelastizität des Gefäßsystems nicht durch Vasopressoren beeinflusst werden kann. 


\section{CME-Fragen}

Under pressure - Der Stellenwert des zentralen Venendrucks in der modernen Intensivmedizin

\section{0}

Der ZVD kann als Marker einer Rechtsherzbelastung oder Herzinsuffizienz herangezogen werden. Welche Aussage dazu ist richtig?
A Eine Linksherzinsuffizienz kann keinen Anstieg des ZVD verursachen.

B Ursache eines erhöhten ZVD bei Rechtsherzinsuffizienz ist eine Diskrepanz zwischen Blutvolumen und potenzieller Kapazität des Gefäßsystems.

C Der ZVD ist bei kardiovaskulären Erkrankungen nicht als prognostischer Marker geeignet.

D Aus einem Anstieg des ZVD kann sicher auf die Progredienz einer Rechtsherzinsuffizienz geschlossen werden.

E Ein erniedrigtes Blutvolumen und ein Abfall des Füllungsdrucks sind initiale Kompensationsmechanismen bei Herzinsuffizienz. 